\title{
Electrically Injected Cavity Polaritons
}

\author{
L. Sapienza, ${ }^{1,2}$ A. Vasanelli,,${ }^{1,2, *}$ R. Colombelli, ${ }^{3}$ C. Ciuti,,${ }^{1,2}$ Y. Chassagneux,${ }^{3}$ \\ C. Manquest, ${ }^{1,2}$ U. Gennser, ${ }^{4}$ and C. Sirtori ${ }^{1,2}$ \\ ${ }^{1}$ Laboratoire "Matériaux et Phénomènes Quantiques", Université Paris Diderot-Paris 7, 75013 Paris, France \\ ${ }^{2}$ CNRS, UMR 7162, 75013 Paris, France \\ ${ }^{3}$ Institut d'Electronique Fondamentale, Université Paris Sud, CNRS, UMR 8622, 91405 Orsay, France \\ ${ }^{4}$ Laboratoire de Photonique et Nanostructures, LPN-CNRS, Route de Nozay, 91460 Marcoussis, France
}

(Received 24 September 2007; published 4 April 2008)

\begin{abstract}
We have realized an electroluminescent device operating in the light-matter strong-coupling regime based on a GaAs/AlGaAs quantum cascade structure embedded in a planar microcavity. At zero bias, reflectivity measurements show a polariton anticrossing between the intersubband transition and the cavity mode. Under electrical injection the spectral features of the emitted light change drastically, as electrons are resonantly injected in a reduced part of the polariton branches. Our experiments demonstrate that electrons can be selectively injected into polariton states up to room temperature.
\end{abstract}

DOI: 10.1103/PhysRevLett.100.136806

The study of electron-hole excitations strongly coupled to photonic modes in a semiconductor microcavity, has motivated a wealth of fascinating experiments [1-3] in the past ten years. Recently, the strong light-matter interaction has been observed, by means of reflectance spectroscopy, also in a two-dimensional electron gas (2DEG) coupled to a cavity photon mode [4-6]. The corresponding lightmatter excitations, called intersubband (ISB) polaritons, have also been probed by photovoltaic measurements [7].

The strong-coupling regime may be especially interesting for light emitting devices (LED) based on ISB transitions. Their radiative efficiency is in fact very poor $\left(\approx 10^{-5}\right)$ because nonradiative phenomena control the lifetime of electrons in excited subbands. In the strong coupling regime the excitation dynamics of the system can be dominated by the vacuum Rabi oscillation frequency rather than by the nonradiative relaxation rate. The possibility of a stimulated emission of ISB polaritons could also lead to inversionless mid- and far-infrared lasers, with lower thresholds with respect to quantum cascade lasers. In the context of light sources, devices based on electrical injection, rather than optical excitation, would be more suitable for applications.

Up to now, electrical injection of a microcavity LED in the strong-coupling regime has only been reported in organic semiconductors [8]. Moreover, the description of a current injecting electrons into a polaritonic system constitutes a new physical framework that lacks a complete theoretical description. A first attempt to describe the coupling between an electronic reservoir and intersubband polaritons has been proposed in Ref. [9].

In the present Letter, we report on the experimental realization of a GaAs/AlGaAs electroluminescent quantum cascade device [10] working in the light-matter strongcoupling regime. Electroluminescence (EL) and absorption spectra measured on the same structure show clear differences, thus pointing out the role of the electrical
PACS numbers: 73.21.Fg, 73.40.Gk, 78.60.Fi, 85.60.Jb

injection. We propose a phenomenological model that accurately reproduces our experimental results.

The sample [sketched in Fig. 1(a)] is composed of a QC LED grown by molecular-beam epitaxy, on an undoped GaAs (001) substrate. The QC LED is placed within a metal/semiconductor planar microcavity, designed for the confinement of transverse magnetic polarized radiation. Light confinement is realized by sandwiching the QC structure between a bottom reflector made of a low refractive index Si-doped $\left(3 \times 10^{18} \mathrm{~cm}^{-3}\right)$ GaAs and $\mathrm{Al}_{0.95} \mathrm{Ga}_{0.05}$ As layers (respectively, 0.56 and

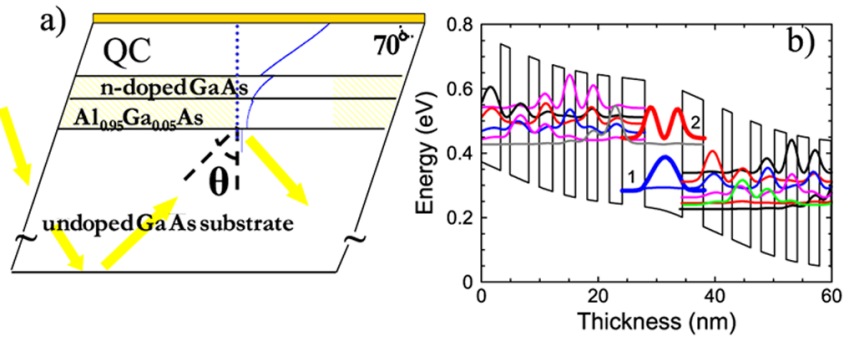

FIG. 1 (color online). (a) Schematic view of the sample. The arrows represent the optical path of the incident beam in the reflectivity measurements; $\theta$ is the propagation angle in the sample. The top and the bottom mirror are shaded. A $86 \mathrm{~nm}$ layer of $1 \times 10^{17} \mathrm{~cm}^{-3} \mathrm{Si}$-doped GaAs and a $17 \mathrm{~nm}$ layer of $3 \times$ $10^{18} \mathrm{~cm}^{-3} \mathrm{Si}$-doped GaAs are grown on top of the QC-LED to allow current flow towards the metallic contacts. The continuous (blue) line represents the intensity of the photon mode along the growth axis and the (blue) dotted line the zero of the mode intensity. (b) Band diagram of the QC-LED for an applied voltage of $6 \mathrm{~V}$. The square moduli of the relevant wave functions are shown. The layer sequence of one period of the structure, in nm, from left to right, starting from the largest well is $6.4 / \mathbf{3 . 6} / 3.3 / \mathbf{1 . 6} / \underline{3.2} / \underline{\mathbf{1 . 8}} / \underline{2.3} / \underline{\mathbf{2 . 0}} / \underline{1.9} / \underline{\mathbf{2 . 0}} / 1.8 / \mathbf{2 . 0} / 2.2 / \mathbf{3 . 9}$. $\mathrm{Al}_{0.45} \mathrm{Ga}_{0.55}$ As layers are in bold, underlined layers are Si doped. This sequence has been repeated 30 times in the sample. 
$0.52 \mu \mathrm{m}$ thick) and a top metallic mirror evaporated on the surface. The latter is also used as top electrical contact.

The band diagram of the QC LED, obtained with selfconsistent Schrödinger-Poisson calculations, is presented in Fig. 1(b) under an applied bias of $6 \mathrm{~V}$. The radiative transition takes place in the largest well between the states labeled 1 and 2, whose nominal energy separation is $E_{12}=$ $160 \mathrm{meV}$. Electrical injection into the subband 2 is obtained by resonant tunnelling through an injection barrier [11]. Electrons are extracted from subband 1 through the states of a miniband, which also inject electrons into the following period. The injection region has been designed in order to have a tunneling time out of the subband 1 longer than the scattering time from 2 to 1 in order to avoid population inversion and accumulate electrons into the subband 1 . This is an essential feature for the observation of the strong-coupling regime [10]. Furthermore, the injection region has been highly doped $\left(7 \times 10^{11} \mathrm{~cm}^{-2}\right)$ in order to provide a high density $2 \mathrm{DEG}$ in the well. As a consequence, the sample is also suitable for reflectivity measurements when no bias is applied [12].

A photon confined within the microcavity can be absorbed promoting an electron to level 2, with the creation of an ISB excitation on top of the 2DEG. By varying the propagating angle of the light within the cavity, the energy of the photonic mode can be tuned across the energy of the ISB excitation. If the strong-coupling regime is achieved, the degeneracy of the excitation and photonic states at resonance will be removed and the two branches will anticross.

The sample has been prepared with $70^{\circ}$ polished facets [see Fig. 1(a)] and a metallic layer $[\operatorname{Ti}(10 \mathrm{~nm}) /$ $\mathrm{Au}(500 \mathrm{~nm})]$ was $e$-beam evaporated on the top surface, for standard reflectivity measurements. Angle resolved reflectivity spectra, performed at $78 \mathrm{~K}$, are shown in Fig. 2. Two peaks are clearly visible across a very wide angular range (between $\approx 59^{\circ}$ and $\approx 80^{\circ}$, where the reflectivity of the facet is approximately constant). At the lowest angles, the two peaks are very close to the cavity mode and to the intersubband transition, whose widths are, respectively, $15 \mathrm{meV}$ and $8 \mathrm{meV}$. In the inset of Fig. 2 we have plotted the energy position of the two peaks (symbols) as a function of the internal angle in the cavity, showing an anticrossing feature at approximately $71^{\circ}$. We attribute the two branches in the inset of Fig. 2 to the upper and lower polaritons (UP and LP, respectively). Because of the steep increase of the upper polariton branch at $\approx 78^{\circ}$, in the last two spectra of Fig. 2 the UP peaks are broadened by the angular resolution of our optical system [13].

The strong-coupling regime has also been observed in reflectivity up to $300 \mathrm{~K}$ with very similar features to those shown in Fig. 2. In the inset of Fig. 2 we have also plotted the LP and UP branches calculated in the transfer matrix formalism (line). The contribution of the intersubband transition has been taken into account in the dielectric

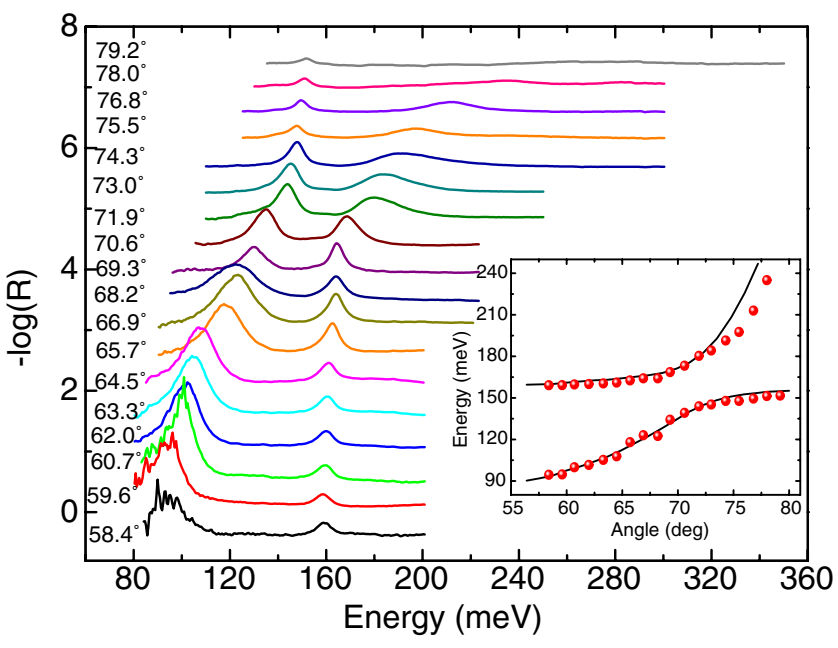

FIG. 2 (color online). Reflectivity spectra as a function of the incident angle, at $78 \mathrm{~K}$ and zero voltage. The spectra result from the ratio $R$ between spectra collected with TM and TE-polarized incident light and are offset each other for clarity. A Nicolet Fourier-transform infrared spectrometer, in rapid scan mode, with a spectral resolution of $8 \mathrm{~cm}^{-1}$, has been used. A $\mathrm{f} / 10.9$ optical system has been used for collection. In the inset, the energy position of the reflectivity peaks is plotted as a function of the angle (full dots), as well as the results of transfer matrix calculations (line).

permittivity of the quantum-well layers including an additional term in the form of an ensemble of classical polarized Lorentz oscillators, as in Ref. [4]. The dispersions of the $\mathrm{Au}$ [14] and of the doped layers [15] have also been included. In the calculations we used an electronic density in the fundamental subband $N_{2 \mathrm{DEG}}=6 \times 10^{11} \mathrm{~cm}^{-2}$ to reproduce the experimental results. This value is consistent with the results of Shubnikov-de Haas measurements which give $7.2 \times 10^{11} \mathrm{~cm}^{-2}$.

The energy splitting deduced from the inset of Fig. 2 is $34 \mathrm{meV}$. It is worth noticing that this value is not the vacuum field Rabi splitting $2 \hbar \Omega_{R}$, which has to be deduced from the $E$ vs $k$ dispersion relation, where $k$ is the in-plane momentum of the photon. The relationship between $k$ and the light propagating angle $\theta$ reads: $k=n E \sin \theta /(c h)$ [16], where $n$ is the substrate refractive index and $E$ the energy of the considered excitation branch. This relationship introduces a strong distortion of the polariton branches, resulting in a value of the Rabi splitting $2 \hbar \Omega_{R}=11 \mathrm{meV}$.

By applying an external bias to the device, the intersubband strong-coupling regime can be investigated via electroluminescence measurements. The sample is thus mesa etched (circular $220 \mu \mathrm{m}$ diameter mesas), metallic contacts $[\mathrm{Ni}(10 \mathrm{~nm}) / \mathrm{Ge}(60 \mathrm{~nm}) / \mathrm{Au}(120 \mathrm{~nm}) / \mathrm{Ni}(20 \mathrm{~nm}) /$ $\mathrm{Au}(200 \mathrm{~nm})]$ are evaporated on top to allow current flow and the facet is polished with an angle of $70^{\circ}$. The sample is soldered onto a copper holder, mounted onto the cold finger of a cryostat and the temperature is varied from 10 to $300 \mathrm{~K}$. The electrical and optical characteristics of the 
device at 78 and $300 \mathrm{~K}$ are summarized in Fig. 3(a), where the evolution of the voltage and of the EL intensity as functions of the injected current density is shown. The structure presents a diodelike behavior, with an alignment at $5 \mathrm{~V}$ at $78 \mathrm{~K}$. The output power shows a well-defined linear dependence with the injected current. The EL spectra have been obtained by applying to the structure a voltage close to the alignment voltage [the working conditions for obtaining the spectra are shown by dots in Fig. 3(a)], in order to keep the electronic density in the lowest subband as high as possible.

Figure 3(b) presents the electroluminescence spectra measured at $78 \mathrm{~K}$ for an applied voltage of $5 \mathrm{~V}$ (left side) and at $300 \mathrm{~K}$ for an applied voltage of $2.8 \mathrm{~V}$ (right side) at different internal angles. The spectral features show a very strong angular dependence. For the angles close to $70^{\circ}$ we can observe at both temperatures two peaks, which are quite well separated in energy. On the
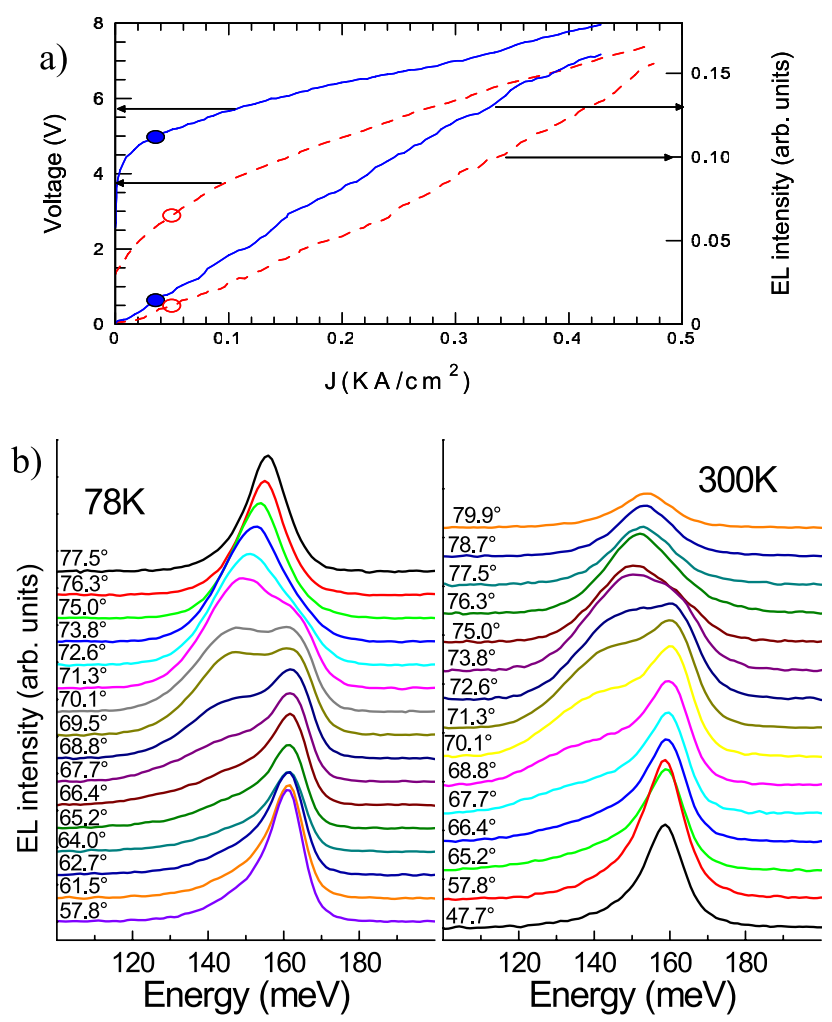

FIG. 3 (color online). (a) Voltage and EL intensity as a function of the injected current density in pulsed operation $(20 \mathrm{kHz}$, $500 \mathrm{~ns}$ ) at $73.8^{\circ}$ at $78 \mathrm{~K}$ (continuous line) and $300 \mathrm{~K}$ (dashed line). The dots indicate the working conditions for obtaining the electroluminescence spectra shown below. (b) EL spectra, offset each other for clarity, as functions of the propagating angle within the microcavity, at $78 \mathrm{~K}$ and $5 \mathrm{~V}$ (left side) and $2.8 \mathrm{~V}$ (right side). These voltages have been chosen as the lowest possible values to measure a spectrum at the two temperatures. In order to increase the EL signal, we worked at $50 \%$ duty cycle (100 kHz repetition rate and $5 \mu$ s pulse width). Spectra are collected in step scan mode, with a spectral resolution of $16 \mathrm{~cm}^{-1}$. contrary, when moving away from the resonance, only one peak is observed with a large shoulder.

The electroluminescence spectra here presented are substantially different from the results obtained in reflectivity: it is important to underline that in reflectivity the polaritons are visible from about 90 to $240 \mathrm{meV}$, while in electroluminescence spectra the energy range where the experimental features are observable is approximately restricted within 120 and $170 \mathrm{meV}$. Furthermore, this energy interval is different in the two sets of data shown in Fig. 3 and depends on the voltage applied to the structure and on the temperature. In order to highlight this point, we show in Fig. 4(a) a comparison between reflectivity and electroluminescence spectra measured at $78 \mathrm{~K}$. In the top panel, the spectra at $65^{\circ}$ are presented. In reflectivity, we observe the UP and LP peaks, while the electroluminescence spectrum shows a single peak close to the energy of the upper polariton with a shoulder towards lower energies. In the lower panel, spectra at $70.1^{\circ}$ are presented: we observe the presence of two peaks in electroluminescence, with an energy difference of about $13 \mathrm{meV}$, a value much lower than the energy difference between the reflectivity peaks (34 meV).

The substantial difference between electroluminescence and reflectivity measurements originate from the coupling that the electronic component of the polariton states has with the injection region. In fact, for a fixed voltage, only one of the states of the injection region has a relevant probability to tunnel through the injection barrier, thus populating the polariton states. In order to simulate the electroluminescence spectra, we describe the injector as a Gaussian function $G(E)=\exp \left[-\left(E-E_{0}\right)^{2} /\left(2 \sigma^{2}\right)\right]$, where $E_{0}$ is related to the energy position of the injector state with respect to the subband $1 ; \sigma$ reflects the broadening of the states in the injector due to interface disorder
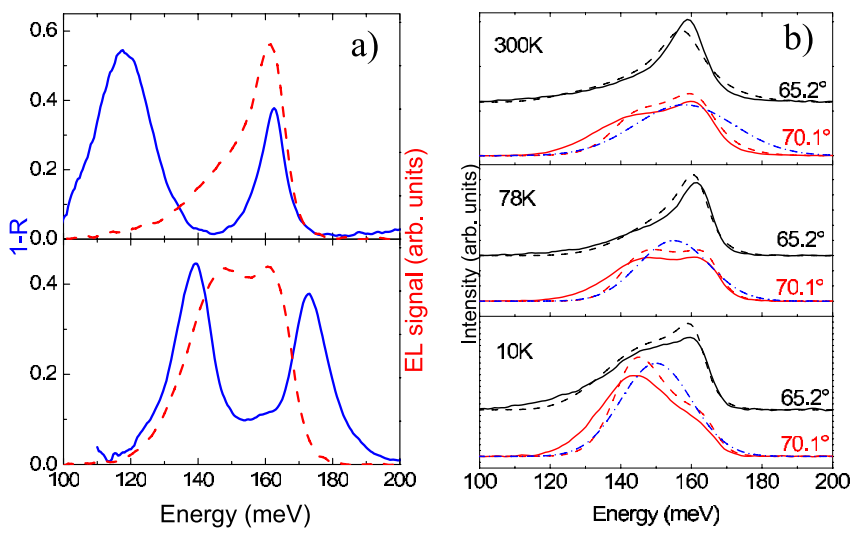

FIG. 4 (color online). (a) Comparison between $1-R$ (i.e., absorption, see text) and EL spectra measured at $78 \mathrm{~K}$, at $65^{\circ}$ (upper panel, reflectivity $65.7^{\circ}$; EL $65.2^{\circ}$ ) and at $70^{\circ}$ (lower panel: reflectivity $70.6^{\circ}$, EL $70.1^{\circ}$ ). (b) Comparison between the experimental (solid lines) and simulated (dashed lines) EL. The dash-dotted (blue) lines indicate the function $G(E)$. 
and impurity scattering [17]. After simulating the absorption spectrum by using the transfer matrix formalism (obtained as $1-R$, by neglecting the transmission through the upper mirror), we calculate the electroluminescence spectrum as the product between the Gaussian function and the absorption spectrum: $S(E)=G(E)(1-R(E))$. The parameters of the Gaussian function, $E_{0}$ and $\sigma$, are kept as fit parameters and determined by the comparison between the simulated and the measured electroluminescence spectra. We show in Fig. 4(b) the measured (solid lines) and simulated (dashed lines) electroluminescence, for three different temperatures (and voltages), at the same angles as in Fig. 4(a). The function $G(E)$ used to reproduce the experimental data is shown in the figure by a dash-dotted (blue) line. We reproduce very well the shapes of the measured spectra in the entire angular range in the three temperature and voltage regimes. The energy position of $G(E)$ has been found to be respectively $E_{0}=150 \mathrm{meV}$ $(10 \mathrm{~K}, 5.2 \mathrm{~V}), E_{0}=155 \mathrm{meV}(78 \mathrm{~K}, 5 \mathrm{~V}), E_{0}=158 \mathrm{meV}$ $(300 \mathrm{~K}, 2.8 \mathrm{~V})$. In the three cases, $E_{0}$ is close to the energy of the ISB transition $\left(E_{12}=160 \mathrm{meV}\right.$ at 10 and $78 \mathrm{~K}$, $E_{12}=156 \mathrm{meV}$ at $\left.300 \mathrm{~K}\right)$. The reason for the differences in the values of $E_{0}$ is due to a different alignment of the states of the injector determined by the different applied bias. A good agreement with the experimental data is found with $\sigma=10 \mathrm{meV}$ for 10 and $78 \mathrm{~K}$, and $\sigma=$ $14 \mathrm{meV}$ for $300 \mathrm{~K}$. In the simulations of the absorption spectrum, we used an electronic density reduced by a factor of $3\left(N_{2 \mathrm{DEG}}=2 \times 10^{11} \mathrm{~cm}^{-2}\right)$ as compared to the unbiased system; this value has been determined by a calculation of the electronic population as a function of the electric field applied to the structure [18] and validated by a comparison between the simulated and measured electroluminescence spectra.

The phenomenological model described above can also be understood in the input-output theoretical framework developed in Ref. [9]. An analytical relationship between the electroluminescence and the absorption spectra may be found by exploiting the unitarity property of the inputoutput matrix [9] to describe the coupling of the system with an electronic reservoir. In this formalism it can be shown that the electroluminescence spectrum takes the form: $\mathcal{L}(E) \propto \mathcal{A}(E) I_{\mathrm{exc}}^{\mathrm{el}}(E)$, where $\mathcal{A}(E)$ is the microcavity absorption spectrum and $I_{\mathrm{exc}}^{\mathrm{el}}(E)$ is related to the spectral properties of the electronic reservoir. This expression is analogue to the one used to interpret our experimental results [19]. The very good agreement between the simulated and the measured spectra of Fig. 4 is a strong proof of the observation of polaritonic luminescence, based on a resonant electrical injection, up to room temperature.

In conclusion, we have realized a semiconductor electroluminescent device based on the light-matter strongcoupling regime. Electroluminescence measurements show an electrical injection into polariton states up to room temperature. Our experimental results have been interpreted by using a phenomenological model, in which an energy filter related to the electrical injection is introduced. We believe that these results open the way to a new class of devices operating in the strong-coupling regime. In the future, an optimized electronic resonant injection into the polariton branches could pave the way to the realization of an electrically pumped polariton laser.

The authors thank I. Carusotto, H.C. Liu, I. Sagnes, N. Alayli, L. Largeau, O. Mauguin, S. De Liberato, and Y. Todorov for fruitful discussions and help. The device fabrication has been performed at the nano center CTUIEF-Minerve, which was partially funded by the "Conseil Général de l'Essonne." We gratefully acknowledge support from EU No. MRTN-CT-2004-51240 POISE and No. ANR-05-NANO-049 INTERPOL.

*Angela.Vasanelli@univ-paris-diderot.fr

[1] C. Weisbuch et al., Phys. Rev. Lett. 69, 3314 (1992).

[2] P. G. Savvidis et al., Phys. Rev. Lett. 84, 1547 (2000).

[3] J. Kasprzak et al., Nature (London) 443, 409 (2006).

[4] D. Dini et al., Phys. Rev. Lett. 90, 116401 (2003).

[5] A. A. Anappara et al., Appl. Phys. Lett. 87, 051105 (2005).

[6] A. A. Anappara et al., Appl. Phys. Lett. 89, 171109 (2006).

[7] L. Sapienza et al., Appl. Phys. Lett. 90, 201101 (2007).

[8] J. R. Tischler et al., Phys. Rev. Lett. 95, 036401 (2005).

[9] C. Ciuti and I. Carusotto, Phys. Rev. A 74, 033811 (2006).

[10] R. Colombelli et al., Semicond. Sci. Technol. 20, 985 (2005).

[11] C. Sirtori et al., IEEE J. Quantum Electron. 34, 1722 (1998).

[12] For the band diagram at zero bias see Ref. [7].

[13] The angular resolution of our system has been estimated to be $\pm 0.8^{\circ}$. This value gives a broadening of $\approx 2 \mathrm{meV}$ on the LP peak at $58^{\circ}$ and of $18 \mathrm{meV}$ on the UP peak at $78^{\circ}$.

[14] M. A. Ordal et al., Appl. Opt. 22, 1099 (1983).

[15] Handbook of Optical Constants of Solids, edited by E. D. Palik (Academic, New York, 1998).

[16] C. Ciuti, G. Bastard, and I. Carusotto, Phys. Rev. B 72, 115303 (2005).

[17] J. Faist et al., Appl. Phys. Lett. 65, 94 (1994).

[18] A. Leuliet (private communication).

[19] A more detailed theoretical description is in progress. 